\title{
Langwirksame Insuline im direkten Vergleich
}

Der direkte Vergleich der beiden langwirksamen Insuline - Insulin glargin $300 \mathrm{E} / \mathrm{ml}$ und Insulin degludec $100 \mathrm{E} / \mathrm{ml}$ - ergab keinen Unterschied hinsichtlich Wirksamkeit. Doch bei der Titration traten unter Insulin glargin $300 \mathrm{E} / \mathrm{ml}$ deutlich weniger Hypoglykämien auf.

Veranstaltung:

Pressekonferenz ${ }_{\text {,Our }}$

focus on improving

outcomes for people

with diabetes now

and for the future",

im Rahmen des EASD

$2018,02.10 .2018$

in Berlin

Veranstalter:

Fa. Sanofi

Autor: Dr. Peter

Stiefelhagen (sti),

Starnberg
Sowohl Insulin glargin $300 \mathrm{E} /$ $\mathrm{ml}\left(\right.$ Toujeo $\left.^{\oplus}\right)$ als auch Insulin degludec $100 \mathrm{E} / \mathrm{ml}$ sind Basalinsuline der zweiten Generation. Das pharmakologische Profil beider Substanzen zeichnet sich durch eine flache Wirkkurve mit lang anhaltender Wirkung aus. Beide Substanzen garantieren eine vergleichbare Wirksamkeit im Hinblick auf die Abnahme des $\mathrm{HbA}_{1 c}$-Wertes und es treten bei Typ-2-Diabetikern unter beiden Insulinen im Vergleich zu Insulin glargin $100 \mathrm{E} / \mathrm{ml}$ weniger Hypoglykämien auf, d. h. beide Insuline bieten eine größere Sicherheit.

\section{BRIGHT-Studie: Head-to-Head- Vergleich}

Im Rahmen der BRIGHT-Studie wurden diese beiden langwirksamen Insuline erstmals direkt miteinander verglichen und zwar sowohl hinsichtlich Wirksamkeit als auch Sicherheit. Es handelt sich dabei um eine multizentrische, offene, randomisierte Studie mit insgesamt 929 Typ-2-Diabetikern, bei denen die Erkrankung mindestens ein Jahr bekannt war. Die Therapie mit einem oralen Antidiabetikum oder einen GLP1-Analogon wurde fortgeführt. Bei Insulin glargin $300 \mathrm{E} / \mathrm{ml}$ wurde die Therapie mit $0,2 \mathrm{U} / \mathrm{kg}$ gestartet, bei Insulin degludec $100 \mathrm{E} / \mathrm{ml}$ betrug die Initialdosis $10 \mathrm{U}$. Die Auftitration erfolgte in den ersten 12 Wochen, wobei ein Glukose-Nüchternwert von 80-100 mg/dL angestrebt wurde.

\section{Weniger Hypoglykämien \\ bei der Titration}

"Im Hinblick auf die Wirksamkeit ergab sich kein Unterschied", so die Studienautorin Professor Chen Alice Cheng von der Abteilung für Endokrinologie in Toronto/Kanada. Unter Insulin glargin $300 \mathrm{E} / \mathrm{ml}$ sank der $\mathrm{HbA}_{1 \mathrm{c}}$-Wert nach einer 24-wöchigen Therapie von 8,7 \% auf 7,0\%, unter Insulin degludec $100 \mathrm{E} /$ $\mathrm{ml}$ von $8,6 \%$ auf $7,0 \%$.

Doch bei der Sicherheit, genauer gesagt bei der Hypoglykämierate, zeigte sich ein deutlicher Unterschied.
Denn eine schwere Hypoglykämie (Glukosewert $<54 \mathrm{mg} / \mathrm{dL}$ ) trat in den ersten 12 Wochen im Rahmen der Titration bei $11,7 \%$ unter Insulin degludec 100 $\mathrm{E} / \mathrm{ml}$, aber nur bei $7,8 \%$ unter Insulin glargin $300 \mathrm{E} / \mathrm{ml}$ auf. Während unter Insulin glargin $300 \mathrm{E} / \mathrm{ml}$ 0,49 schwere Hypoglykämien pro Patientenjahr dokumentiert wurden, waren es unter Insulin degludec $100 \mathrm{E} / \mathrm{ml}$ 0,86 Ereignisse pro Patientenjahr. Insgesamt war die Hypoglykämie-Inzidenz in der Titrationsphase bei Insulin glargin E 300/ ml um $37 \%$ niedriger. In der abschließenden stabilen Behandlungsphase gab es zwischen den beiden Substanzen keinen Unterschied bei den Hypoglykämien.

J. Klin. Endokrinol. Stoffw. 2019 • 12:38 https://doi.org/10.1007/s41969018-0045-4

(c) Springer-Verlag GmbH Austria, ein Teil von Springer Nature 2018

Hier steht eine Anzeige. 